\title{
Return to the Origin as a Probe of Atomic Phase Coherence
}

\author{
Clément Hainaut, ${ }^{1}$ Isam Manai, ${ }^{1}$ Radu Chicireanu, ${ }^{1}$ Jean-François Clément, ${ }^{1}$ Samir Zemmouri, ${ }^{1}$ Jean \\ Claude Garreau, ${ }^{1}$ Pascal Szriftgiser, ${ }^{1}$ Gabriel Lemarié, ${ }^{2}$ Nicolas Cherroret, ${ }^{3}$ and Dominique Delande ${ }^{3}$ \\ ${ }^{1}$ Université de Lille, CNRS, UMR 8523 - PhLAM - Laboratoire de \\ Physique des Lasers Atomes et Molécules, F-59000 Lille, France* \\ ${ }^{2}$ Laboratoire de Physique Théorique, UMR 5152, \\ CNRS and Université de Toulouse, F-31062 Toulouse, France \\ ${ }^{3}$ Laboratoire Kastler Brossel, UPMC-Sorbonne Universités, CNRS, \\ ENS-PSL Research University, Collège de France, 4 Place Jussieu, 75005 Paris, France
}

\begin{abstract}
We report on the observation of the coherent enhancement of the return probability ("enhanced return to the origin", ERO) in a periodically kicked cold-atom gas. By submitting an atomic wave packet to a pulsed, periodically shifted laser standing wave, we induce an oscillation of ERO in time and explain it in terms of a periodic, reversible dephasing in the weak-localization interference sequences responsible for ERO. Monitoring the temporal decay of ERO, we exploit its quantum coherent nature to quantify the decoherence rate of the atomic system.
\end{abstract}

PACS numbers: 03.75.-b , 72.15.Rn, 05.45.Mt, 64.70.qj

The transport of waves in disordered or chaotic systems can be strongly affected by interference effects, with striking signatures for both quantum and classical waves: coherent backscattering, universal conductance fluctuations [1], Anderson localization [2] and its many-body counterpart [3]. Intuitively, one expects multiple scattering by disorder to lead to a pseudo-random walk, and to an average diffusive behavior at long time. For waves however, the situation is quite different: even at moderate disorder strengths spectacular manifestations of localization can already show up. A well known example is weak localization. In time-reversal invariant systems, two paths counterpropagating on a closed loop have the same amplitude and phase; they interfere constructively, doubling the probability to return to the starting point.

Because weak localization crucially relies on timereversal symmetry and phase coherence, it has been exploited in many contexts to probe decoherence or magnetic field effects. In particular, in mesoscopic electronic systems, it features a reduction of the diffusion coefficient and constitutes an invaluable asset for probing the electronic phase coherence [4-6]. In classical wave systems, weak localization is usually evidenced by the coherent backscattering effect, which corresponds to an enhanced probability for a wave to be reflected from a disordered medium in the backward direction [7-10]. A third consequence of weak localization is the enhancement of the probability that an expanding wave packet returns to its origin ("enhanced return to the origin", ERO). This effect manifests itself as a narrow peak visible at the center of the density profile of the wave packet. ERO has been observed in classical wave systems, for instance in the nearfield intensity profile of seismic waves propagating in the crust [11] or of acoustic waves in chaotic cavities [12, 13].

Recent cold atom experiments [14] offer a high level of control on crucial ingredients like statistical properties of disorder, dimensionality, interactions and coupling to the environment. This has led to clear new observations of Anderson localization [15, 16], coherent backscattering [17], and recently many-body localization [18]. On
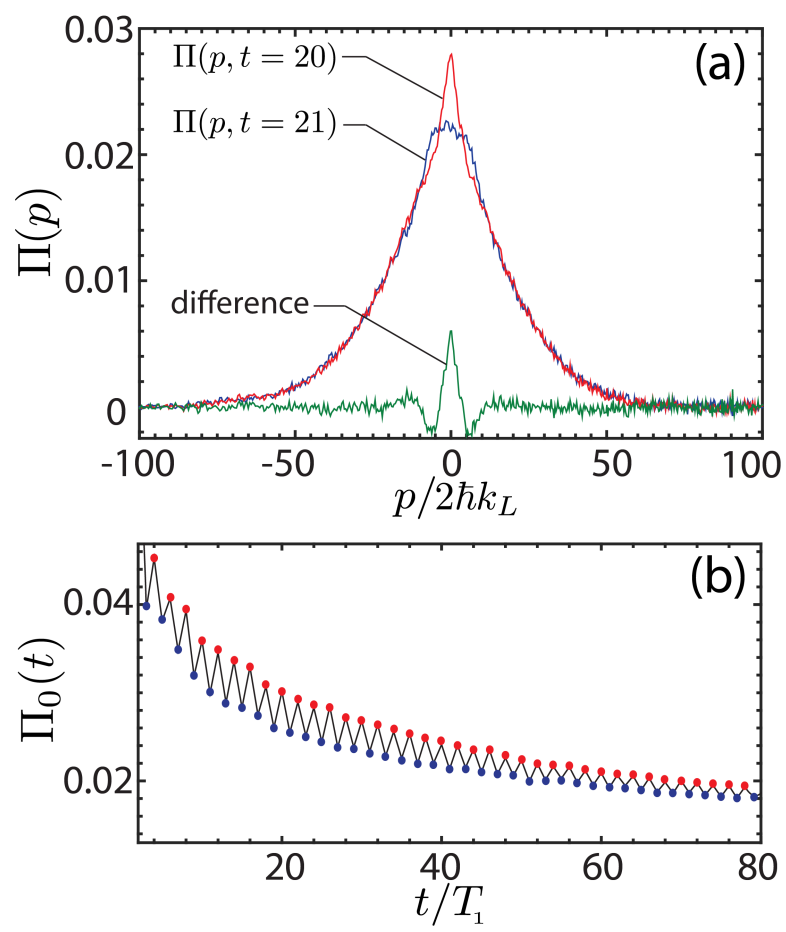

Figure 1. (Color online) Experimental observation of enhanced return to the origin. (a) Momentum distribution $\Pi(p, t)$ at an even $(t=20$, red curve) and an odd ( $t=21$, blue curve) kick. The distribution around $p=0$ at $t=20$ is enhanced with respect to the distribution at $t=21$, as evidenced by the green difference signal. (b) The zero-momentum population $\Pi_{0}$ vs. $t$ shows a clear oscillation between even kicks (ERO, red dots) and odd kicks (blue dots). The attenuation in the contrast is due to decoherence. Parameters are $K=12$, $\hbar=1.5$ and $a=0.04$. 
the other hand, the atomic quantum kicked rotor (QKR), a model system for quantum chaos [19], has played a key role in the observation of dynamical localization, a suppression of the classical chaotic diffusion in momentum space by quantum interference [20,21], analog to Anderson localization [22]. By adding modulation frequencies [23, 24], "quantum simulations" [25] of multidimensional Anderson models have been realized in 2D [26] and 3D [27-31] systems, where the metal-insulator transition has been completely characterized.

Experimentally, ERO is difficult to observe as it requires an initially narrow wave packet and a good spatial resolution. In this Letter, we use the full control of the scattering events (here the kicks) that occur during the propagation of the atomic kicked rotor - in contrast with usual disordered media where scattering events occur randomly in time - to periodically trigger or extinguinsh the interference mechanisms at the origin of ERO. The observation of ERO is achieved through striking oscillations of the return probability. It thus constitutes a excellent probe of the "building blocks" of the interference processes leading to localization. Furthermore, by following in time the destruction of ERO, we measure the decoherence of the system, in the spirit of studies conducted in mesoscopic physics. Decoherence is nowadays recognized as a fundamental process bridging quantum physics at the microscopic scale with classical physics at the macroscopic scale $[32,33]$.

In our experiment, a cloud of laser-cooled atoms is exposed to a pulsed, far-detuned standing wave (SW). A key feature is the use of a modified version of the QKR [34], in which the SW is spatially shifted every second kick by an amount $a$. We call such a system "periodically-shifted QKR" (PSQKR), and it is described by the Hamiltonian

$H=\frac{p^{2}}{2}+K \sum_{n}[\cos x \delta(t-2 n)+\cos (x+a) \delta(t-2 n+1)]$

where time is measured in units of the SW pulse period $T_{1}$, space in units of $\left(2 k_{L}\right)^{-1}$ with $k_{L}=2 \pi / \lambda_{L}$ the laser wave number, and momenta in units of $2 \hbar k_{L}$ such that $[x, p]=i \times 4 \hbar k_{L}^{2} T_{1} / M=i \hbar$, defining the reduced Planck constant $k . K$ is proportional to the intensity and to the inverse of the detuning of the SW. Note that, for $a=0$, Eq. (1) reduces to the Hamiltonian of the usual QKR [20].

For the kicked rotor, diffusion and localization take place in momentum space, hence ERO will manifest itself as a narrow peak around the initial momentum $p \approx 0$ in the momentum density. Its observation thus requires a very good momentum resolution, both in the measurement and in the preparation processes. The experimental ERO signal is convoluted with the width of the initial momentum distribution, which reduces the enhancement factor well below the expected value of 2 , making its direct observation difficult. It is thus necessary to start with a momentum distribution as narrow as possible. We load Cs atoms in a standard Magneto-Optical Trap (MOT), and cool them further by an optimized molasses phase, which cools the atoms to a temperature of $2 \mu \mathrm{K}$.

We then apply a pulsed optical standing wave [35], formed by two independent laser beams [26]. The standing wave is spatially shifted by changing the phase of one beam with respect to the other; doing so each other kick realizes the PSQKR described by the Hamiltonian (1). As this Hamiltonian is of period 2, the ERO peak is present only each second kick (see below), making its observation easier (see Fig. 1).

The atomic momentum distribution $\Pi(p, t)$ is detected by a standard time-of-flight technique at the end of the sequence. At even kicks (to which no spatial shift is applied) we clearly observe an enhancement of $\Pi(p)$ in the vicinity of $p=0$ [red curve in Fig. 1(a)] for $t=20$. In contrast, at odd kicks $[t=21$, blue curve in Fig. 1(a)] no enhancement is visible. Fig. 1(b) shows $\Pi_{0}(t) \equiv \Pi(p=0, t)$; one sees that this oscillatory behavior persists up to long times $t>80$.

One can understand the origin of the oscillation of ERO in our system by considering the PSQKR evolution operator over one time period (corresponding to two kicks). For symmetry reasons, we choose to consider the evolution operator $U$ from time $2 n-1 / 2$ to $2 n+3 / 2$. Indeed, momentum densities do not evolve during free propagation between kicks, so the final results do not depend on the origin of time. This evolution operator can then be split in a "shifted" (odd) kick operator $U_{a}$ and a "non-shifted" (even) evolution operator $U_{0}: U=U_{a} U_{0}$ with

$$
\begin{aligned}
& U_{a}=\exp \left(-\frac{i \hat{p}^{2}}{4 k}\right) \exp [-i \kappa \cos (\hat{x}+a)] \exp \left(-\frac{i \hat{p}^{2}}{4 k}\right) \\
& U_{0}=\exp \left(-\frac{i \hat{p}^{2}}{4 k}\right) \exp [-i \kappa \cos \hat{x}] \exp \left(-\frac{i \hat{p}^{2}}{4 k}\right)
\end{aligned}
$$

where $\kappa \equiv K / \hbar$. A key point for ERO is the existence of constructive interference between time-reversed paths. In the usual QKR, this is due to the invariance of the evolution operator over one kick - which coincides with $U_{0}$ - under the generalized time-reversal symmetry operator $\mathcal{T}=T P$, product of the time-reversal anti-unitary operator $T: t \rightarrow-t$ with the unitary parity operator $P: x \rightarrow-x$, such that $\mathcal{T}: t \rightarrow-t ; x \rightarrow-x ; p \rightarrow p$ preserves momentum. For the PSQKR, $\mathcal{T}=T P$ is not a symmetry operation, because the $a$ term in $U_{a}$ is not parity-invariant. However, the product $\mathcal{T}_{a}=T P_{a / 2}$ of the time-reversal operator by the parity operator with respect to $a / 2, P_{a / 2}: x \rightarrow a-x$ exchanges $U_{0}$ and $U_{a}$ : $\mathcal{T}_{a} U_{0, a} \mathcal{T}_{a}=U_{a, 0}$ Thus, for even numbers of kicks the symmetry is preserved: $\mathcal{T}_{a}\left(U_{a} U_{0}\right)^{n} \mathcal{T}_{a}=\left(U_{a} U_{0}\right)^{n}$, but, for odd numbers of kicks, an orphaned $U_{0}$ or $U_{a}$ operator remains, breaking the symmetry. As a consequence, multiple scattering paths which are images of each other by 

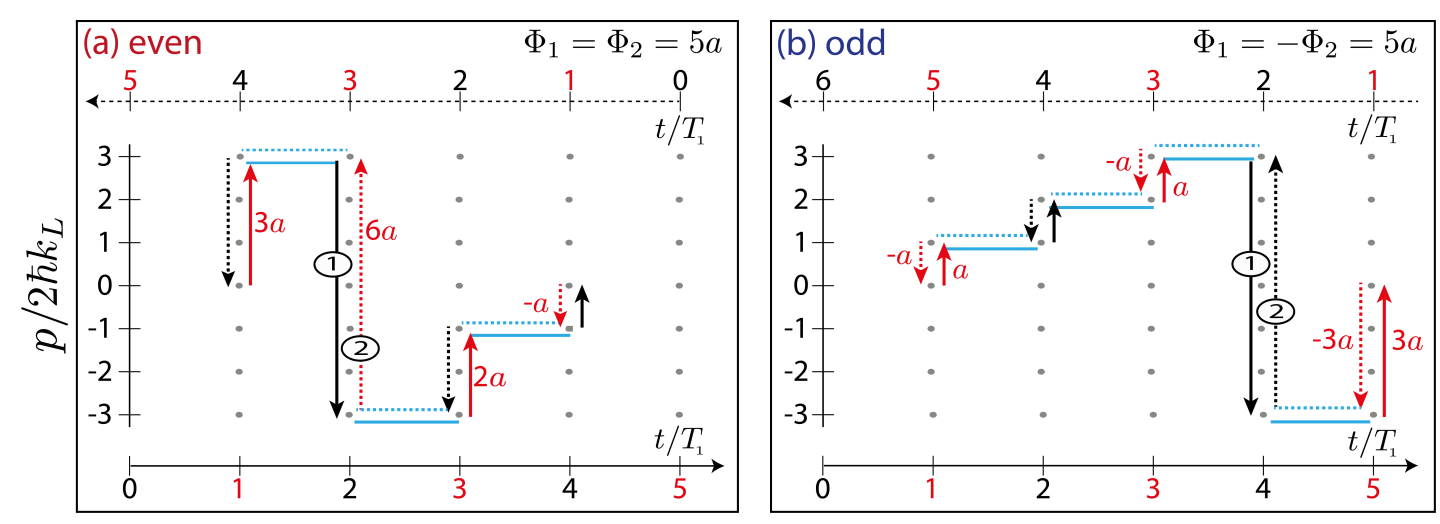

Figure 2. (Color online) Paths in momentum space at the origin of ERO in the PSQKR. For each path we show the amplitude (solid lines) and its time-reversed associate (dashed lines). Kick momentum transfers are represented by vertical arrows, red for odd kicks and black for even kicks. Arrows point in the direction of increasing time. Horizontal lines symbolize free propagation between kicks. (a) Paths involving four kicks: Path 1 accumulates an $a$-dependant phase $\Phi_{1}=3 a+2 a=5 a$ associated with the shifted SW position at first and third kicks. The time-reversed path 2 accumulates exactly the same phase $\Phi_{2}=-a+6 a=5 a$, making ERO visible. (b) Same thing for five kicks: The total phases are respectively $a+a+3 a=5 a$ and $-a-a-3 a=-5 a$. The phase difference is $\Delta \Phi=10 a \neq 0$ and the ERO is suppressed.

$\mathcal{T}_{a}$ will accumulate the same phase, leading to a constructive interference, very much like time-reversed paths are responsible for weak localization in usual time-reversal invariant disordered systems.

To illustrate this reasoning, let us consider an example. With periodic boundary conditions [36] along $x$, we can use the eigenbasis associated with the $\hat{p}$ operator, labeled by an integer $n$ such that $\hat{p}|n\rangle=n k|n\rangle$. The free propagation operator in this basis is diagonal, while the kick operator is $\exp [-i \kappa \cos (\hat{x}+a)]=\sum_{m}(-i)^{m} J_{m}(\kappa) \mathrm{e}^{i m a} \mid n+$ $m\rangle\langle n|$ (with $a=0$ for even kicks). For odd kicks $(a \neq 0)$ the side bands generated from component $n$ get an additional phase $m a$, where $m$ is the change in momentum. In panel (a) of Fig. 2 we represent by a broken solid line a "momentum path" (labeled 1) involving 4 kicks, to which we match the associated time-reversed path 2 (broken dashed line). Such sequence of counter-propagating paths is responsible for ERO [37]. One sees that both the direct and the time-reversed paths accumulate the same phase (here $\Phi_{1}=\Phi_{2}=5 a$ ). The dephasing $\Phi_{1}-\Phi_{2}$ vanishes, making ERO visible. In contrast, considering a 5 kick path and its time-reversed image, Fig. 2b, a residual dephasing $\left(\Phi_{1}-\Phi_{2}=10 a\right)$ remains, suppressing ERO.

The periodic manifestation of ERO in our system can also be understood from the diagrammatic technique [38]. Assuming that transport is supported by diffusion, we find

$$
\Pi_{0}(t) \simeq \frac{1}{\sqrt{4 \pi D t}}\left[1+e^{-\Gamma t} \times\left\{\begin{array}{cc}
1 & \text { if } t \text { even } \\
\mathrm{e}^{-a^{2} D t} & \text { if } t \text { odd }
\end{array}\right]\right.
$$

where $D$ is the diffusion coefficient and $\Gamma$ the decoherence rate of the system. The second term in the square brackets is the contribution of ERO. In agreement with the experimental observation, at finite $a$ this contribution is strongly suppressed at odd kicks. While Eq. (4) predicts an enhancement factor of 2 between even and odd kicks for sufficiently large $a$, the experimentally observed factor is significantly lower, essentially due to the convolution with the initial momentum profile as discussed above. Note also that the $t^{-1 / 2}$ dependance of the ERO signal is expected to be valid only in the initial diffusion stage, whereas the decay at long times is essentially dominated by exponential terms in Eq. (4) (see Figure 4).

To demonstrate that the experimental ERO signal is due to quantum interference between pairs of closed loops, we add a controlled amount of decoherence to the system. For this purpose, we define the quantity $\Delta_{t}=(-1)^{t}\left[\Pi_{0}(t=n)-\Pi_{0}(t=n-1)\right]$, the difference of the zero-momentum population between two successive kicks. We shine on the atoms a resonant laser ("decoherer") beam at $t=21^{+}$(i.e just after the $21^{\text {st }}$ kick) of a PSQKR sequence, thus producing spontaneous emissioninduced decoherence. The decoherer is applied during $20 \mu \mathrm{s}$ (up to $t=23$ ) and its intensity is adjusted to produce an average number $N_{\mathrm{sp}}$ of spontaneous emission events per atom. This number is independently calibrated by shining the decoherer beam on the MOT cloud and measuring the radiation pressure force it exerts on the atomic sample. The effect of the decoherer beam on the ERO signal is shown in Fig. 3: the oscillating behavior of $\Pi_{0}$ is rapidly quenched after kick 21 , which proves the coherent nature of the observed ERO. The inset of Fig. 3 shows the decrease of $\Delta_{t=28} v s . N_{\mathrm{sp}}$, displaying an exponential behavior $\exp \left(-N_{\mathrm{sp}}\right)$. Indeed, ERO still exists after the decoherer pulse, due to atoms which have not scattered any resonant photon, and, as this is a Poissonian process, the probability of scattering zero photon is $\exp \left(-N_{\mathrm{sp}}\right)$. The remaining small $\Delta_{t=28}$ at large $N_{\mathrm{sp}}$ is probably due to the incomplete quenching of phase coherence by spontaneous emission. 


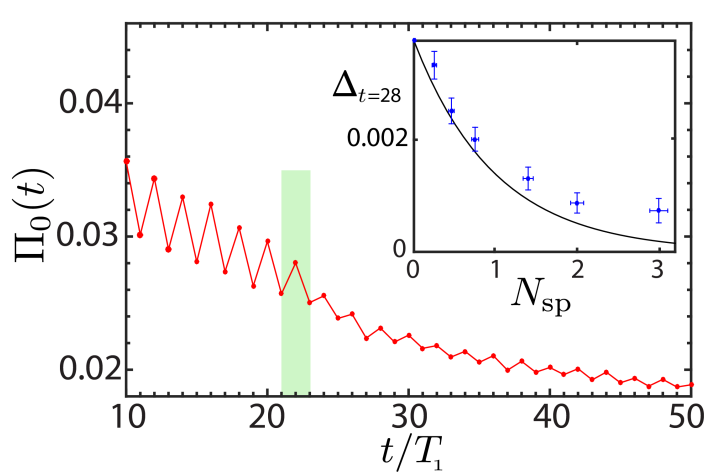

Figure 3. (Color online) Zero-momentum probability density $\Pi_{0}$ vs. $t$ for the PSQKR. A decoherer beam is applied between the $21^{\text {st }}$ and $23^{\text {rd }}$ pulses (green-shadowed region), quenching the oscillations. Parameters are $K=12, k=1.5$ and $a=$ 0.04. The decoherer beam induces an average of $N_{\mathrm{sp}}=2$ spontaneous emission events per atom. The inset shows the reduction in the difference signal $\Delta_{t}$ as a function of $N_{\mathrm{sp}}$; the black line is the expected exponential decay $\exp \left(-N_{\mathrm{sp}}\right)$ (it is not a fit).

The ERO signal can also be used to measure the amount of decoherence present in the system. We observe an exponential decay of $\Delta_{t} v s$. $t$, shown in the inset of Fig. 4, from which one can determine the decoherence rate $\Gamma_{0}: \Gamma_{0}=0.024$ for $K=12$ and $\Gamma_{0}=0.014$ for $K=9$. Which physical mechanisms induce this decoherence is presently unknown [39]. We can nevertheless test the reliability of the method by applying the decoherer beam during the whole experimental sequence, thus introducing a controlled amount of spontaneous emission. The beam intensity, calibrated in situ by measuring the radiation pressure on the atomic cloud as described above, is chosen to produce a controlled decoherence rate $\Gamma_{\text {ext }}$. From the decay of $\Delta_{t} v s$. $t$, we determine the total decoherence rate $\Gamma$. We expect the latter to be given by $\Gamma=\Gamma_{\text {ext }}+\Gamma_{0}$. The straight line of slope 1 in Fig. 4 (not a fit) proves that it is indeed the case, so that we have a reliable measurement of decoherence rates, very much like magnetoconductance is used in solid state physics to measure the electronic phase coherence length [4-6].

In conclusion, we have experimentally observed the phenomenon of enhanced return to the origin with atomic matter waves, a clear signature of weak localization in time-reversal invariant systems. By controlling the phase of the scattering events induced by the standing wave kicks, we have induced a time-periodic oscillation of ERO, allowing for a clear observation of its contrast. A crucial ingredient is the ability to control precisely the even/odd number of scattering events, a unique advantage of the kicked rotor, in contrast with ordinary disordered systems where only the average number of scattering events is under control. Finally, by introducing a controlled amount of decoherence, we have verified its

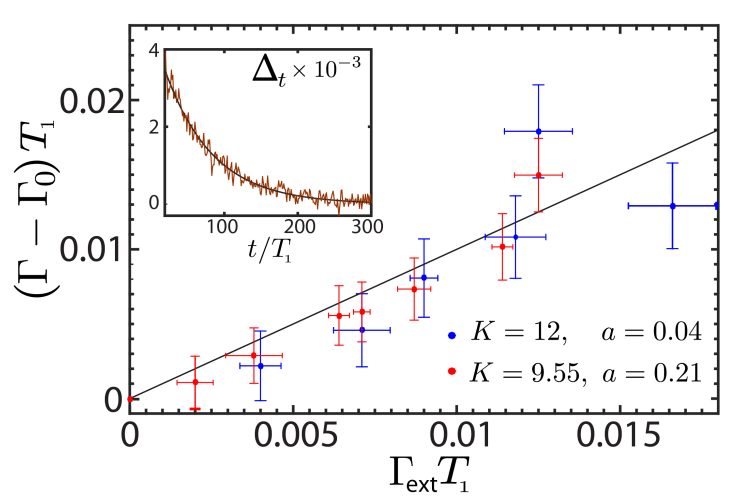

Figure 4. (Color online) Probing decoherence with ERO. Inset: the decay of the difference signal $\Delta_{t} v s$. $t$ is fitted by an exponential (black line) from which the decoherence rate $\Gamma$ is extracted. In the absence of any externally applied decoherence, this gives the $-K$ and $a$ dependent - "stray" decoherence rate $\Gamma_{0}$. This procedure is repeated in the presence of the decoherer beam for several values of the additional imposed decoherence rate $\Gamma_{\text {ext }}$. The fact that the excess rate $\Gamma-\Gamma_{0}$ measured using the decay of the ERO signal agrees perfectly with the externally added rate $\Gamma_{\text {ext }}$ shows that ERO is a faithful measure of decoherence.

quantum nature and used it to access the decoherence rate in the system. This work opens promising perspectives in the use of coherent phenomena to probe sources of decoherence in atomic systems, as well as other sources of dephasing such as interactions [40]. Phase control of scattering events may also constitute an alternative approach to artificial gauge fields [41] to induce effective magnetic field effects in cold atom systems.

The authors are grateful to C. Tian for fruitful discussions. This work is supported by Agence Nationale de la Recherche (Grants LAKRIDI No. ANR-11-BS040003-02 and K-BEC No. ANR-13-BS04-0001-01), the Labex CEMPI (Grant No. ANR-11-LABX-0007-01), and "Fonds Européen de Développement Economique Régional" through the "CPER Photonics for Society (P4S)". This work was granted access to the HPC resources of TGCC under the allocation 2016-057083 made by GENCI (Grand Equipement National de Calcul Intensif)

* www.phlam.univ-lille1.fr/atfr/cq

[1] E. Akkermans and G. Montambaux, Mesoscopic physics of electrons and photons (Cambridge University Press, 2007).

[2] E. Abrahams, 50 years of Anderson Localization, Vol. 24 (World Scientific, 2010).

[3] D.M. Basko, I.L. Aleiner, and B.L. Altshuler, "Metalinsulator transition in a weakly interacting many-electron system with localized single-particle states," Annals of physics 321, 1126-1205 (2006). 
[4] G. Bergman, "Influence of Spin-Orbit Coupling on Weak Localization," Phys. Rev. Lett. 48, 1046-1049 (1982).

[5] Y. Niimi, Y. Baines, T. Capron, D. Mailly, F.-Y. Lo, A. D. Wieck, T. Meunier, L. Saminadayar, and C. Bäuerle, "Effect of Disorder on the Quantum Coherence in Mesoscopic Wires," Phys. Rev. Lett. 102, 226801 (2009).

[6] T. Capron, C. Texier, G. Montambaux, D. Mailly, A. D. Wieck, and L. Saminadayar, "Ergodic versus diffusive decoherence in mesoscopic devices," Phys. Rev. B 87, 041307 (2013).

[7] P.-E. Wolf and G. Maret, "Weak Localization and Coherent Backscattering of Photons in Disordered Media," Phys. Rev. Lett. 55, 2696-2699 (1985).

[8] M. P. V. Albada and A. Lagendijk, "Observation of Weak Localization of Light in a Random Medium," Phys. Rev. Lett. 55, 2692-2695 (1985).

[9] G. Labeyrie, F. Tomasi, J. C. Bernard, C. A. Muller, C. Miniatura, and R. Kaiser, "Coherent backscattering of light by cold atoms," Phys. Rev. Lett. 83, 5266-5269 (1999).

[10] G. Labeyrie, D. Delande, R. Kaiser, and C. Miniatura, "Light transport in cold atoms and thermal decoherence," Phys. Rev. Lett. 97, 013004 (2006).

[11] E. Larose, L. Margerin, B. A. van Tiggelen, and M. Campillo, "Weak Localization of Seismic Waves," Phys. Rev. Lett. 93, 048501 (2004).

[12] J. de Rosny, A. Tourin, and M. Fink, "Coherent Backscattering of an Elastic Wave in a Chaotic Cavity," Phys. Rev. Lett. 84, 1693-1695 (2000).

[13] R. L. Weaver and O. I. Lobkis, "Enhanced Backscattering and Modal Echo of Reverberant Elastic Waves," Phys. Rev. Lett. 84, 4942-4945 (2000).

[14] L. Sanchez-Palencia and M. Lewenstein, "Disordered quantum gases under control," Nat. Phys. 6, 87-95 (2010).

[15] J. Billy, V. Josse, Z. Zuo, A. Bernard, B. Hambrecht, P. Lugan, D. Clément, L. Sanchez-Palencia, P. Bouyer, and A. Aspect, "Direct observation of Anderson localization of matter-waves in a controlled disorder," Nature (London) 453, 891-894 (2008).

[16] G. Roati, C. d'Errico, L. Fallani, M. Fattori, C. Fort, M. Zaccanti, G. Modugno, M. Modugno, and M. Inguscio, "Anderson localization of a non-interacting BoseEinstein condensate," Nature (London) 453, 895-898 (2008).

[17] F. Jendrzejewski, K. Müller, J. Richard, A. Date, T. Plisson, P. Bouyer, A. Aspect, and V. Josse, "Coherent Backscattering of Ultracold Atoms," Phys. Rev. Lett. 109, 195302 (2012).

[18] M. Schreiber, S.S. Hodgman, P. Bordia, H.P Lüschen, M.H Fischer, R. Vosk, E. Altman, U. Schneider, and I. Bloch, "Observation of many-body localization of interacting fermions in a quasirandom optical lattice," Science 349, 842-845 (2015).

[19] F. M. Izrailev, "Simple models of quantum chaos: spectrum and eigenfunctions," Phys. Rep. 196, 299-392 (1990).

[20] G. Casati, B. V. Chirikov, J. Ford, and F. M. Izrailev, "Stochastic behavior of a quantum pendulum under periodic perturbation," in Stochastic Behavior in Classical and Quantum Systems, Vol. 93, edited by G. Casati and J. Ford (Springer-Verlag, Berlin, Germany, 1979) pp. $334-352$.
[21] F. L. Moore, J. C. Robinson, C. F. Bharucha, B. Sundaram, and M. G. Raizen, "Atom Optics Realization of the Quantum $\delta$-Kicked Rotor," Phys. Rev. Lett. 75, 4598-4601 (1995).

[22] S. Fishman, D. R. Grempel, and R. E. Prange, "Chaos, Quantum Recurrences, and Anderson Localization," Phys. Rev. Lett. 49, 509-512 (1982).

[23] D. L. Shepelyansky, "Localization of diffusive excitation in multi-level systems," Physica D 28, 103-114 (1987).

[24] G. Casati, I. Guarneri, and D. L. Shepelyansky, "Anderson Transition in a One-Dimensional System with Three Incommensurate Frequencies," Phys. Rev. Lett. 62, 345348 (1989).

[25] I. M. Georgescu, S. Ashhab, and F. Nori, "Quantum simulation," Rev. Mod. Phys. 86, 153-185 (2014).

[26] I. Manai, J.-F. Clément, R. Chicireanu, C. Hainaut, J. C. Garreau, P. Szriftgiser, and D. Delande, "Experimental Observation of Two-Dimensional Anderson Localization with the Atomic Kicked Rotor," Phys. Rev. Lett. 115, 240603 (2015).

[27] J. Chabé, G. Lemarié, B. Grémaud, D. Delande, P. Szriftgiser, and J. C. Garreau, "Experimental Observation of the Anderson Metal-Insulator Transition with Atomic Matter Waves," Phys. Rev. Lett. 101, 255702 (2008).

[28] G. Lemarié, H. Lignier, D. Delande, P. Szriftgiser, and J. C. Garreau, "Critical State of the Anderson Transition: Between a Metal and an Insulator," Phys. Rev. Lett. 105, 090601 (2010).

[29] M. Lopez, J.-F. Clément, P. Szriftgiser, J. C. Garreau, and D. Delande, "Experimental Test of Universality of the Anderson Transition," Phys. Rev. Lett. 108, 095701 (2012).

[30] M. Lopez, J.-F. Clément, G. Lemarié, D. Delande, P. Szriftgiser, and J. C. Garreau, "Phase diagram of the anisotropic Anderson transition with the atomic kicked rotor: theory and experiment," New J. Phys 15, 065013 (2013).

[31] G. Lemarié, J. Chabé, P. Szriftgiser, J. C. Garreau, B. Grémaud, and D. Delande, "Observation of the Anderson metal-insulator transition with atomic matter waves: Theory and experiment," Phys. Rev. A 80, 043626 (2009).

[32] W. H. Zurek, "Decoherence, einselection, and the quantum origins of the classical," Rev. Mod. Phys. 75, 715775 (2003).

[33] S. Haroche, "Nobel Lecture: Controlling photons in a box and exploring the quantum to classical boundary," Rev. Mod. Phys. 85, 1083-1102 (2013).

[34] C. Tian, A. Kamenev, and A. Larkin, "Ehrenfest time in the weak dynamical localization," Phys. Rev. B 72, 045108 (2005).

[35] In practice the kicks have a finite duration $\tau=350 \mathrm{~ns}$. Free propagation can be neglected as long as the atom motion during this time is small compared to the scale of variation of the potential, that is $v \tau \ll \lambda_{L} / 2$, where $v$ is the atom velocity and $\lambda_{L}$ the wavelength of the standing wave.

[36] In the experiment, the system is extended along $x$ so that we cannot use periodic boundary conditions. Nevertheless, the system is invariant by a $2 \pi$ spatial translation, so that the Bloch theorem applies. Any initial state of the system can be written as a linear combination of different quasimomenta $\beta k$ in the first Brillouin zone $-1 / 2<\beta \leq 1 / 2$. The various $\beta$ components are un- 
coupled and evolve independently. $\beta$ - like the momentum itself - is preserved by the $\mathcal{T}$ symmetry, so that all $\beta$ components display the ERO phenomenon. The only change is the replacement $n \rightarrow n+\beta$ for the phase accumulated during free propagation, which affects similarly the pair of conjugate paths.

[37] V. N. Prigodin, B. L. Altshuler, K. B. Efetov, and S. Iida, "Mesoscopic dynamical echo in quantum dots," Phys. Rev. Lett. 72, 546-549 (1994).

[38] A. Altland, "Diagrammatic approach to Anderson localization in the quantum kicked rotator," Phys. Rev. Lett.
71, 69-72 (1993).

[39] See Ref. [31] for a more complete discussion of the possible decoherence sources in our setup.

[40] M. Hartung, T. Wellens, C. A. Müller, K. Richter, and P. Schlagheck, "Coherent Backscattering of Bose-Einstein Condensates in Two-Dimensional Disorder Potentials," Phys. Rev. Lett. 101, 020603 (2008).

[41] J. Dalibard, F. Gerbier, G. Juzeliūnas, and P. Öhberg, "Artificial gauge potentials for neutral atoms," Rev. Mod. Phys. 83, 1523-1543 (2011). 